\title{
A narrative review of current and potential prognostic biomarkers for immunotherapy in small-cell lung cancer
}

\author{
Jeong Uk Lim ${ }^{1}$, Hye Seon Kang ${ }^{2}$ \\ ${ }^{1}$ Division of Pulmonary, Critical Care and Allergy, Department of Internal Medicine, Yeouido St. Mary's Hospital, College of Medicine, The \\ Catholic University of Korea, Seoul, Republic of Korea; ${ }^{2}$ Division of Pulmonary, Critical Care and Allergy, Department of Internal Medicine, \\ Bucheon St. Mary's Hospital, College of Medicine, The Catholic University of Korea, Seoul, Republic of Korea \\ Contributions: (I) Conception and design: JU Lim; (II) Administrative support: HS Kang; (III) Provision of study materials or patients: All authors; \\ (IV) Collection and assembly of data: All authors; (V) Data analysis and interpretation: JU Lim; (VI) Manuscript writing: Both authors; (VII) Final \\ approval of manuscript: Both authors. \\ Correspondence to: Hye Seon Kang. Division of Pulmonary, Critical Care and Allergy, Department of Internal Medicine, Bucheon St. Mary's \\ Hospital, College of Medicine, The Catholic University of Korea, 327, Sosa-ro, Bucheon-si, Gyeonggi-do 14647, Republic of Korea. \\ Email: beyer_kr@catholic.ac.kr.
}

\begin{abstract}
Small-cell lung cancer (SCLC) is a highly invasive and rapidly proliferating pathologic subtype that accounts for $13-15 \%$ of all lung cancer cases. Recently in extensive-stage SCLC, treatments that combine immunotherapy and chemotherapy showed increased efficacy compared to chemotherapy alone in several trials. However, the combination of immunotherapy and conventional chemotherapy regimens was introduced only recently for extensive-stage SCLC, with relatively little real-world data. The demand for reliable biomarkers that can predict the efficacy of immunotherapy in SCLC is high. Several studies evaluated various parameters including programmed cell death ligand-1 (PD-L1) expression, tumor mutation burden (TMB), gene expression profiling, autoantibody, and blood cytokines for predictive value for response to immunotherapy in SCLC. Despite some observed correlations, there is a lack of concrete support for the use of PD-L1 expression levels for readily available biomarker. High TMB in combination with smoking history is predictive of a better response to immunotherapy, but validation of cutoffs and testing methods is necessary before it can be widely applied in clinical settings. Other candidate biomarkers such as immune cell distribution among tumor microenvironment, and systemic inflammatory markers can also be evaluated, after an accumulation of real-life data from SCLC patients under immunotherapy.
\end{abstract}

Keywords: Small-cell lung cancer (SCLC); immunotherapy; biomarker; tumor

Submitted Jan 06, 2021. Accepted for publication Mar 24, 2021.

doi: $10.21037 /$ atm-21-68

View this article at: http://dx.doi.org/10.21037/atm-21-68

\section{Background}

Small-cell lung cancer (SCLC) is a highly invasive and rapidly proliferating pathologic subtype that accounts for $13-15 \%$ of all lung cancer cases $(1,2)$. SCLC is much more likely to develop in smokers than in non-smokers (3). About two-thirds of patients who are initially diagnosed with SCLC develop extensive-stage SCLC (ES-SCLC) (4). The prognosis for ES-SCLC is poor, with a median overall survival of $8-13$ months (5). For the past two decades, etoposide in combination with either carboplatin or cisplatin has been the standard first-line regimen for ESSCLC $(6,7)$. Although response rates to this regimen have reached $60-65 \%$, the median overall survival (OS) is only 10 months $(8,9)$.

Immune cells, including cytotoxic T-cells, contain programmed cell death protein $1(\mathrm{PD}-1)$ and cytotoxic T-lymphocyte associated protein 4 (CTLA-4). When programmed death-ligand 1 (PD-L1) on the surface of cancer cells binds to receptors on cytotoxic T-cells, immune 
responses against tumor cells are inhibited. Studies of immune evasion by cancer cells have led to significant advances in the development of immunotherapies that block PD-1 and CTLA-4 on T cells from binding to PD-L1 on cancer cells $(10,11)$.

Treatments that combine immunotherapy and chemotherapy can increase immune responses to tumor cells, resulting in increased efficacy compared to chemotherapy alone (11). Several recent trials have combined immunotherapy targeting the programmed cell death-1 (PD-1)/programmed cell death ligand-1 (PDL1) pathway to a platinum-based regimen, resulting in improved overall survival (OS) in patients with ES-SCLC $(12,13)$. The IMpower 133 and CASPIAN studies showed similar results, collectively suggesting a breakthrough in the treatment of SCLC. The addition of atezolizumab to the traditional etoposide-carboplatin regimen, followed by atezolizumab maintenance until progression, has shown a superior median OS of 12.3 months compared to 10.3 months in the placebo group, and a median progression free-survival (PFS) of 5.2 months compared to 4.3 months in the placebo group (12). In patients with advanced SCLC who were treated in CheckMate 032, the 2 -year overall survival rate was $26 \%$ among those treated with nivolumab plus ipilimumab, compared to $14 \%$ in the nivolumab monotherapy group (14). This treatment was particularly enhanced in patients with high tumor mutation burden (TMB) (15). The combination of durvalumab and platinum-based regimens also showed promising results $(13,16)$. However, the combination of immunotherapy and platinum-based chemotherapy regimens was introduced only recently, with relatively little real-world data. It is likely that not all patients with ES-SCLC would benefit from this combination treatment. Even though the impact of adding immunotherapy is modest in improving OS, it is beneficial to search for biomarker to predict clinical outcomes and personalize cancer treatment. Therefore, the demand for reliable biomarkers that can predict the efficacy of immunotherapy in SCLC is high, and efforts to identify predictive markers are ongoing.

In this mini-review, we assess and discuss biomarkers evaluated in previous studies of immunotherapy in SCLC, as well as other candidate markers that may predict clinical outcomes.

We present the following article in accordance with the Narrative Review reporting checklist (available at http:// dx.doi.org/10.21037/atm-21-68).

\section{Method}

\section{Search strategy}

Using the Preferred Reporting Items for Systematic Reviews and Meta-analyses (PRISMA) guidelines (17), an online search of literature on biomarkers for efficacy of immunotherapy in ES-SCLC was conducted. The National Center for Biotechnology Information (NCBI) PubMed, Cochrane Library, EMBASE and Google Scholar were searched. All literature published in English between January 2014 and November 2020 were included. Various combinations of search texts were used. Search word 'SCLC' or 'small cell lung' was combined with each of the following terms; 'immunotherapy', 'immune checkpoint', 'nivolumab', 'atezolizumab', 'pembrolizumab', 'durvalumab', 'ipilimumab', 'biomarker', 'marker', 'prognosis', 'programmed cell death ligand-1', 'PD-L1', and 'tumor mutation burden'.

\section{Current immunotherapy regimens in ES-SCLC}

\section{First line therapy}

After decades without major breakthrough, immune checkpoint inhibitors (ICIs) are the notable agents to show an improvement in outcomes of patients with ES-SCLC. Clinical trials of single and combination regimens show some significant results.

The phase III study IMpower133 showed that a combination regimen including immunotherapy as firstline treatment improved both OS and PFS. A total of 403 treatment-naïve patients with ES-SCLC were assigned to carboplatin plus etoposide with either atezolizumab or placebo, followed by atezolizumab or placebo maintenance. The median OS was significantly longer in the atezolizumab group than the placebo group (12.3 vs. 10.3 months; HR 0.70, 95\% CI: 0.54-0.91; $\mathrm{P}=0.007)$. The median PFS was 5.2 and 4.3 months, respectively (HR $0.77,95 \% \mathrm{CI}$ : $0.62-0.96, \mathrm{P}=0.02)(12)$. The efficacy of ipilimumab in combination with carboplatin and etoposide as first-line therapy for ES-SCLC was also tested in both phase II and phase III studies (CA184-041, NCT01331525, and CA184-156), and the results suggested the possibility of a significant breakthrough. Even though median OS was not different between chemotherapy plus ipilimumab group and chemotherapy plus placebo group, median PFS was longer in the chemotherapy plus ipilimumab group (4.6 vs. 4.4 months, HR 0.85; 95\% CI: 0.75-0.97, P=0.0161) 
(18-20). The phase III CASPIAN study showed that the addition of durvalumab to a combination regimen of etoposide with either cisplatin or carboplatin (EP) significantly improved OS compared with EP alone in patients with treatment-naïve ES-SCLC (13.0 months vs. 10.3 months, HR 0.73; 95\% CI: 0.59-0.91, $\mathrm{P}=0.0047)(13)$.

\section{Second or higher line therapy}

Several studies which evaluated the efficacy of immunotherapy for recurrent SCLC have been performed. In the Checkmate 032 study in which patients were eligible regardless of tumor PD-L1 expression, double checkpoint inhibitor combination of nivolumab and ipilimumab, and nivolumab monotherapy demonstrated anti-tumor activity with durable responses in previously treated patients with SCLC. Objective response rate (ORR) was 10\% (95\% CI: $5-18 \%)$ in the nivolumab monotherapy group and $23 \%$ (95\% CI: $13-36 \%$ ) in the combination group (21). However, median OS was similar between the groups (4.7, 95\% CI: $3.1-8.3$ vs. $5.7,95 \%$ CI: 3.8-7.6) (14). Based on these data, FDA granted an accelerated approval to nivolumab for the treatment of metastatic refractory SCLC in August 2018. Furthermore, a randomized phase III trial (CheckMate 331) was conducted to evaluate the efficacy of nivolumab compared to a standard second-line treatment with either topotecan or amrubicin upon investigator's decision in patients with relapsed SCLC following platinum-based chemotherapy, however, nivolumab did not show survival benefit when compared to the conventional chemotherapy regimen (median OS, 7.5 vs. 8.4 months; HR, 0.86; 95\% CI: 0.72-1.04; $\mathrm{P}=0.11$ ) (22).

Different clinical trials evaluated the efficacy of immunotherapy for refractory SCLC, but they did not show encouraging results. Atezolizumab was evaluated in the IFCT-1,603 non-comparative phase II study and resulted in ORR of $2.3 \%$ and median PFS of 1.4 months (23). In KEYNOTE-028 study, pembrolizumab treatment for PDL1 expressing ( $\geq 1 \%$ ) recurrent SCLC showed ORR of 33\% (95\% CI: $16 \%$ to $55 \%$ ), but limited PFS of 1.9 months (95\% CI: 1.7 to 5.9 months) (24). Also, durvalumab failed to show satisfactory results, ORR of $10 \%$ and PFS of 1.5 months (25).

\section{PD-L1 and PD-1 expression}

One of the immune escape mechanisms of tumor cells is the interaction of PD-L1 with PD-1 on T cells, which inhibits $\mathrm{T}$ cell activation and cytotoxicity (26). The understanding of ICIs is based on the PD-1/PD-L1 axis, and PD-L1 expression has been evaluated as a potential predictive biomarker for ICI efficacy in several studies $(27,28)$. However, despite some observed correlations, there is a lack of concrete support for the use of PD-L1 expression levels in SCLC as a predictive biomarker for immunotherapy response (29). First, the pooled prevalence of PD-L1 expression is lower compared to those reported in non-small cell lung cancer (NSCLC), which are influenced by IHC evaluation cut-off values, PD-L1 staining pattern, the quality of the study's methodological characteristics, specimen acquisition, and other technical problems such as antibody staining and tissue fixation $(14,30,31)$. Second, PD-L1 expression heterogeneity is not fully captured by small piece of tissue. Circulating tumor cells (CTCs) can represent tumor PD-L1 expression on the whole, but technical problem such as CTCs isolation and validation with matched biopsy and resected tumor are remained (32). Although positive PD-L1 expression appears to show better OS in SCLC patients, using it as a reliable predictive marker requires further large scaled studies. In a meta-analysis, the pooled HR of all studies was 0.86 (95\% CI: $0.49-1.50, \mathrm{P}=0.588$ ) indicating that positive PD-L1 expression demonstrated a trend towards longer OS in SCLC patients (14). However, in the Checkmate 032 study, only $17 \%$ of patients had $\geq 1 \%$ PD-L1 expression and $5 \%$ had $\geq 5 \%$ PD-L1 expression, and the response to nivolumab monotherapy or a combination with ipilimumab occurred irrespective of the PD-L1 expression levels (21).

\section{Tumor mutation burden}

Like NSCLC, SCLC is characterized by high TMB, which has been shown to predict the efficacy of nivolumab in NSCLC treatment (33), suggesting a potential significant benefit for TMB as a biomarker for immunotherapy in SCLC. In SCLC cohort of CheckMate 032, nivolumab \pm ipilimumab showed an improved efficacy in patients with high TMB. In this study, TMB was defined as the total number of somatic missense mutations, and patients were divided into tertiles. Tertile boundaries were defined as: low, 0 to $<143$ mutations; medium, 143 to 247 mutations, and high, $\geq 248$ mutations (15). Previous studies on immunotherapy in SCLC have shown that high TMB in combination with smoking history is predictive of a better response to immunotherapy $(31,34)$. However, exploratory subgroup analyses performed in the IMpower 133 study showed that blood-based TMB levels at a cutoff 
of 10 or 16 mutations per megabase had no clear predictive power for atezolizumab response. The authors explained that the highly active and myelosuppressive nature of the combination of platinum and etoposide affected the predictability of blood-based TMB (12). TMB is not readily available as a biomarker for clinical practice yet, because a larger population study is necessary to validate reliable cutoffs and testing methods before it can be widely applied in clinical settings. In addition, tissue acquisition is an important issue in TMB assessment, because many patients with SCLC are likely to receive only small cytology sample biopsies. Therefore, core biopsies are necessary for patients with SCLC in order to perform routine TMB testing (35). Also, clinical use of circulating free DNA (cfDNA) for TMB evaluation instead of tissue sample is needed. Many problems such as low cfDNA concentration in patients with low tumor burden and low variant allelic frequency affecting the test results remain (36).

\section{Targeted gene sequencing and gene expression profiling}

In a multicenter phase II study, 26 patients with ES-SCLC who showed progression after etoposide plus platinum chemotherapy received six cycles of paclitaxel every 3 weeks. Pembrolizumab was added after the first cycle of paclitaxel and maintained until progression. In this study, targeted gene sequencing (TGS) designed to detect 90 cancer-related genes was performed in 14 patients. Favorable survival outcomes were obtained in the group harboring MET copy number gain (PFS 10.5 vs. 3.4 months, $\mathrm{P}=0.019$ ) (37).

A pan-cancer, T-cell-inflamed gene-expression profile (GEP) comprised of 18 genes indicative of a T-cell-activated tumor microenvironment (TME) was associated with pembrolizumab response in multiple cancers. T cell-inflamed TME characterized by active IFN- $\gamma$ signaling, cytotoxic effector molecules, antigen presentation, and T-cell active cytokines, is a common feature of tumor biology that are responsive to PD-1 checkpoint blockade (38). In the phase Ib Keynote 028 trial, which enrolled 475 patients with PD-L1-positive advanced solid tumors, the patient group with SCLC showed an ORR of $33 \%$ following treatment with pembrolizumab. The study showed that tumor T cellinflamed GEPs were a potential predictive biomarker of pembrolizumab response (39). T cell-inflamed GEP scores were higher in patients showing good responses and longer PFS. However, since Keynote 028 examined various solid tumors, a separate study enrolling a large number of SCLC patients should be conducted for validation.

\section{Autoantibodies and cytokines}

Other clinical parameters, such as autoantibody and inflammatory cytokine levels, have been evaluated for their potential values as biomarker. In a multicenter phase 2 study of ipilimumab in combination with carboplatin and etoposide as first-line therapy for ES-SCLC, a positive autoimmune profile at baseline was associated with better PFS according to immune response criteria. Patients with any positive autoantibody detected at baseline showed a significantly longer median PFS [8.8 months (95\% CI: 5.1-10.7) versus 7.3 months (95\% CI: 2.9-7.9), $\mathrm{P}=0.036$ ]. Also, an association between anti-SOX2 and anti-Hu autoantibody levels, and severe neurological toxicities was observed (19).

Predictive values of inflammatory cytokines for the immunotherapy response have been studied in NSCLC (40), and one such study including two cohorts of SCLC patients evaluated the predictive value of cytokines in SCLC patients under immunotherapy. Changes in the levels of various cytokines, including IL-1 $\beta$, IL-2, IL-4, IL-5, IL-6, IL-8, IL-10, and IFN- $\gamma$, were compared between the patients treated with chemotherapy only and those treated with chemotherapy plus ipilimumab. Compared with the chemotherapy-only group, patients treated with ipilimumab showed a global increase of all cytokines after treatment initiation. Increased baseline IL-2 levels were predictive of the ipilimumab response. Patients with a high serum IL-2 concentration had a median OS of 30.5 months while those with lower concentration had a median OS of 8 months $(\mathrm{P}=0.015)$. A serial increase in the IL-4 levels was associated with improved OS in the combination treatment group. Patients whose IL-4 increased more than $32 \%$ had a significantly better OS (18.5 vs. 8.8 months; $\mathrm{P}=0.042)$ (41).

\section{Other candidate biomarkers}

A study of resected samples from long-term survivors (survival $>4$ years) and survivors with the expected survival time (survival $\leq 2$ years) who were diagnosed with SCLC applied quantitative immunostaining to confirm differences in immune cell distribution among tumor microenvironments. The number of tumorinfiltrating and associated lymphocytes was higher 
throughout tumors of long-term survivors than in those of expected survivors with an OS of $<2$ years, irrespective of clinical variables including TNM staging and curativeversus non-curative-intent surgery (42). Considering that immune response activation against tumor cells is the main mechanism of ICIs, the distribution of CD markers which reflect immune cell profiles in the tumor microenvironment may be a potential biomarker.

The lung immune prognostic index (LIPI), which is calculated from the serum lactate dehydrogenase levels and the ratio of derived neutrophils to lymphocytes (dNLR), is also a potential biomarker in SCLC patients under immunotherapy. A study of 171 patients with SCLC who underwent anticancer treatment modalities other than immunotherapy (concurrent chemoradiotherapy, conventional chemotherapy, radiotherapy) showed that LIPI had prognostic value in terms of OS and PFS (43). The authors hypothesized that systemic inflammation may have an adverse effect on the efficacy of chemotherapy, which may be reflected in the LIPI scores. Other systemic inflammatory biomarkers such as NLR and the plateletto-lymphocyte ratio (PLR) may have prognostic value in SCLC patients under immunotherapy, since they are associated with OS or PFS in SCLC patients under conventional anticancer treatment including etoposide combination regimens $(44,45)$. The publication of further studies of SCLC under immunotherapy using systemic inflammatory biomarkers is anticipated following an accumulation of survival data.

An examination of the immune evasion mechanism of tumor cells can provide clues to predicting the immune response to SCLC at the cellular level. The interaction between SCLC tumor cells and T regulatory (Treg) cells, which modulate the activity of other $\mathrm{T}$ cells, may be involved in SCLC immunotherapy. Patients with SCLC showing higher ratios of FOXP3+ cells in tumor infiltrates have been reported to have a poorer prognosis. Some SCLC tumor cell lines induce denovo differentiation of functional FOXP3 + Treg cells in healthy blood lymphocytes. SCLC tumor cells can induce CD4 $\mathrm{T}$ cell-mediated immunosuppression, suggesting a potential mechanism by which SCLC cells downregulate immune responses against tumor cells, in turn leading to poorer survival (46). Downregulation of the major histocompatibility complex (MHC) has also been suggested as a main mechanism of immunotherapy resistance (47). MHC class I expression has been reported to be lower in SCLC tumor samples than in NSCLC cell lines (48), and decreased interferon- $\gamma$ secretion is thought to greatly contribute to the reduction of MHC in SCLC (49).

Finally, the associations between galectin-9 (Gal-9) and the tumor-immune microenvironment and immune infiltration in SCLC patients under concurrent chemoradiotherapy, conventional chemotherapy or radiotherapy, were studied. The Gal-9 expression levels on tumor-infiltrating lymphocytes (TILs) were found to be correlated with the PD-1, PD-L1, CD3, CD4, CD8, and FOXP3 levels, and high Gal-9 protein expression on TILs was correlated with superior recurrence-free survival, suggesting the potential of Gal-9 as a biomarker in SCLC patients under immunotherapy (50). In addition, other biomarkers which had shown potential predictive values in other cancers including NSCLC and skin cancer, and not yet tested in ES-SCLC can also be evaluated. T cell receptor clonality, TILs, blood soluble cytokine concentrations, and immune gene signature had shown association with ICI response in melanoma, breast, and lung cancer (51-53) (Table 1).

\section{Conclusions}

Besides TMB, no reliable biomarker has been discovered that can predict the immunotherapy response in SCLC patients. Future studies focused on understanding of basic biology of SCLC and the identification of novel predictive biomarkers of response to immunotherapy in SCLC are essential. 
Table 1 Predictive biomarkers of anti-PD-1/PD-L1 therapy in SCLC in comparison to NSCLC

\begin{tabular}{|c|c|c|c|c|c|c|}
\hline $\begin{array}{l}\text { Candidate } \\
\text { biomarker }\end{array}$ & \multicolumn{3}{|c|}{ SCLC } & \multicolumn{3}{|c|}{ NSCLC } \\
\hline TMB & - & $\begin{array}{l}\text { Nivolumab } \pm \text { ipilimumab showed } \\
\text { an improved efficacy in patients } \\
\text { with high TMB }\end{array}$ & (15) & - & $\begin{array}{l}\text { Higher TMB associated with } \\
\text { improved clinical response, } \\
\text { clinical benefit, and PFS to } \\
\text { anti-PD-1/PD-L1 treatment }\end{array}$ & $(33,51,54,55)$ \\
\hline Mutation & MET copy gain & $\begin{array}{l}\text { Twenty-six patients who show } \\
\text { progression after } 1^{\text {st }} \text { line } \\
\text { chemotherapy were treated with } \\
\text { pembrolizumab and paclitaxel. } \\
\text { Favorable survival outcomes } \\
\text { obtained in the group of ES-SCLC } \\
\text { harboring MET copy number gain }\end{array}$ & (37) & $\begin{array}{l}\text { MET exon } 14 \\
\text { alterations }\end{array}$ & $\begin{array}{l}\text { A study included } 24 \text { patients } \\
\text { with MET exon } 14 \text {-altered } \\
\text { lung cancers who received } \\
\text { immunotherapy. ORR was } \\
17 \% \text { ( } 95 \% \text { Cl: } 6 \% \text { to } 36 \%) \text {. } \\
\text { The median PFS was } 1.9 \\
\text { months ( } 95 \% \mathrm{Cl}: 1.7-2.7)\end{array}$ & (58) \\
\hline $\begin{array}{l}\text { Immune cell } \\
\text { distribution }\end{array}$ & $\begin{array}{l}\text { T cell-inflamed } \\
\text { GEP scores }\end{array}$ & $\begin{array}{l}\text { In the phase lb Keynote } 028 \\
\text { trial, the patient group with SCLC } \\
\text { showed an ORR of } 33 \% \text { after } \\
\text { treatment with pembrolizumab. } \\
\text { The study showed that tumor T } \\
\text { cell-inflamed GEPs were a } \\
\text { potential predictive biomarker of } \\
\text { pembrolizumab response }\end{array}$ & (39) & $\begin{array}{l}\text { Cytotoxic CD8+ } \\
\text { T cells/TILS }\end{array}$ & $\begin{array}{l}\text { Increase in post-treatment } \\
\text { TILS was associated with } \\
\text { anti-CTLA-4 response } \\
\text { CD8+ TILs in early stage } \\
\text { lung cancer was prognostic } \\
\text { for disease free survival and } \\
\text { overall survival. }\end{array}$ & $(52,59,60)$ \\
\hline
\end{tabular}

IL, interleukin; IFN, interferon; TNF, tumor necrosis factor; PD, programmed death; ICI, immune checkpoint inhibitor; SCLC, small cell lung cancer; NSCLC, non-small cell lung cancer; ES, extensive-stage; PFS, progression-free survival; ORR, objective response rate; OS, overall survival; GEP, gene-expression profile; TMB, tumor mutation burden; Cl, confidence interval; TIL, tumor-infiltrating lymphocytes; CTLA, cytotoxic T-lymphocyte-associated protein 4; MET, mesenchymal-to-epithelial transition.

\section{Acknowledgments}

Funding: None.

\section{Footnote}

Reporting Checklist: The authors have completed the Narrative Review reporting checklist. Available at http:// dx.doi.org/10.21037/atm-21-68

Conflicts of Interest: Both authors have completed the ICMJE uniform disclosure form (available at http://dx.doi. org/10.21037/atm-21-68). The authors have no conflicts of interest to declare.

Ethical Statement: The authors are accountable for all 
aspects of the work in ensuring that questions related to the accuracy or integrity of any part of the work are appropriately investigated and resolved.

Open Access Statement: This is an Open Access article distributed in accordance with the Creative Commons Attribution-NonCommercial-NoDerivs 4.0 International License (CC BY-NC-ND 4.0), which permits the noncommercial replication and distribution of the article with the strict proviso that no changes or edits are made and the original work is properly cited (including links to both the formal publication through the relevant DOI and the license). See: https://creativecommons.org/licenses/by-nc-nd/4.0/.

\section{References}

1. Rudin CM, Ismaila N, Hann CL, et al. Treatment of Small-Cell Lung Cancer: American Society of Clinical Oncology Endorsement of the American College of Chest Physicians Guideline. J Clin Oncol 2015;33:4106-11.

2. Dela Cruz CS, Tanoue LT, Matthay RA. Lung cancer: epidemiology, etiology, and prevention. Clin Chest Med 2011;32:605-44.

3. Ettinger DS, Aisner J. Changing face of small-cell lung cancer: real and artifact. J Clin Oncol 2006;24:4526-7.

4. Oronsky B, Reid TR, Oronsky A, et al. What's New in SCLC? A Review. Neoplasia 2017;19:842-7.

5. Calles A, Aguado G, Sandoval C, et al. The role of immunotherapy in small cell lung cancer. Clin Transl Oncol 2019;21:961-76.

6. Fruh M, De Ruysscher D, Popat S, et al. Small-cell lung cancer (SCLC): ESMO Clinical Practice Guidelines for diagnosis, treatment and follow-up. Ann Oncol 2013;24 Suppl 6:vi99-105.

7. Rudin CM, Giaccone G, Ismaila N. Treatment of SmallCell Lung Cancer: American Society of Clinical Oncology Endorsement of the American College of Chest Physicians Guideline. J Oncol Pract 2016;12:83-6.

8. Farago AF, Keane FK. Current standards for clinical management of small cell lung cancer. Transl Lung Cancer Res 2018;7:69-79.

9. Socinski MA, Smit EF, Lorigan P, et al. Phase III study of pemetrexed plus carboplatin compared with etoposide plus carboplatin in chemotherapy-naive patients with extensivestage small-cell lung cancer. J Clin Oncol 2009;27:4787-92.

10. Pardoll DM. The blockade of immune checkpoints in cancer immunotherapy. Nat Rev Cancer 2012;12:252-64.

11. Konala VM, Madhira BR, Ashraf S, et al. Use of
Immunotherapy in Extensive-Stage Small Cell Lung Cancer. Oncology 2020;98:749-54.

12. Horn L, Mansfield AS, Szczesna A, et al. First-Line Atezolizumab plus Chemotherapy in Extensive-Stage Small-Cell Lung Cancer. N Engl J Med 2018;379:2220-9.

13. Paz-Ares L, Dvorkin M, Chen Y, et al. Durvalumab plus platinum-etoposide versus platinum-etoposide in firstline treatment of extensive-stage small-cell lung cancer (CASPIAN): a randomised, controlled, open-label, phase 3 trial. Lancet 2019;394:1929-39.

14. Acheampong E, Abed A, Morici M, et al. Tumour PDL1 Expression in Small-Cell Lung Cancer: A Systematic Review and Meta-Analysis. Cells 2020;9:2393.

15. Hellmann MD, Callahan MK, Awad MM, et al. Tumor Mutational Burden and Efficacy of Nivolumab Monotherapy and in Combination with Ipilimumab in Small-Cell Lung Cancer. Cancer Cell 2018;33:853-61.e4.

16. Goldman JW, Garassino MC, Chen Y, et al. Patientreported outcomes with first-line durvalumab plus platinum-etoposide versus platinum-etoposide in extensive-stage small-cell lung cancer (CASPIAN): a randomized, controlled, open-label, phase III study. Lung Cancer 2020;149:46-52.

17. Moher D, Liberati A, Tetzlaff J, et al. Preferred reporting items for systematic reviews and meta-analyses: the PRISMA statement. J Clin Epidemiol 2009;62:1006-12.

18. Reck M, Bondarenko I, Luft A, et al. Ipilimumab in combination with paclitaxel and carboplatin as first-line therapy in extensive-disease-small-cell lung cancer: results from a randomized, double-blind, multicenter phase 2 trial. Ann Oncol 2013;24:75-83.

19. Arriola E, Wheater M, Galea I, et al. Outcome and Biomarker Analysis from a Multicenter Phase 2 Study of Ipilimumab in Combination with Carboplatin and Etoposide as First-Line Therapy for Extensive-Stage SCLC. J Thorac Oncol 2016;11:1511-21.

20. Reck M, Luft A, Szczesna A, et al. Phase III Randomized Trial of Ipilimumab Plus Etoposide and Platinum Versus Placebo Plus Etoposide and Platinum in Extensive-Stage Small-Cell Lung Cancer. J Clin Oncol 2016;34:3740-8.

21. Antonia SJ, López-Martin JA, Bendell J, et al. Nivolumab alone and nivolumab plus ipilimumab in recurrent smallcell lung cancer (CheckMate 032): a multicentre, openlabel, phase 1/2 trial. Lancet Oncol 2016;17:883-95.

22. Spigel DR, Vicente D, Ciuleanu TE, et al. SecondLine Nivolumab in Relapsed Small-Cell Lung Cancer: CheckMate 331. Ann Oncol 2021;32:631-41.

23. Pujol JL, Greillier L, Audigier-Valette C, et al. A 
Randomized Non-Comparative Phase II Study of AntiProgrammed Cell Death-Ligand 1 Atezolizumab or Chemotherapy as Second-Line Therapy in Patients With Small Cell Lung Cancer: Results From the IFCT-1603 Trial. J Thorac Oncol 2019;14:903-13.

24. Ott PA, Elez E, Hiret S, et al. Pembrolizumab in Patients With Extensive-Stage Small-Cell Lung Cancer: Results From the Phase Ib KEYNOTE-028 Study. J Clin Oncol 2017;35:3823-9.

25. Goldman JW, Dowlati A, Antonia SJ, et al. Safety and antitumor activity of durvalumab monotherapy in patients with pretreated extensive disease small-cell lung cancer (ED-SCLC). J Clin Oncol 2018;36:8518.

26. Sabari JK, Lok BH, Laird JH, et al. Unravelling the biology of SCLC: implications for therapy. Nat Rev Clin Oncol 2017;14:549-61.

27. Taube JM, Klein A, Brahmer JR, et al. Association of PD1, PD-1 ligands, and other features of the tumor immune microenvironment with response to anti-PD-1 therapy. Clin Cancer Res 2014;20:5064-74.

28. Patel SP, Kurzrock R. PD-L1 Expression as a Predictive Biomarker in Cancer Immunotherapy. Mol Cancer Ther 2015;14:847-56.

29. Esposito G, Palumbo G, Carillio G, et al. Immunotherapy in Small Cell Lung Cancer. Cancers (Basel) 2020;12:2522.

30. Guo H, Li L, Cui J. Advances and challenges in immunotherapy of small cell lung cancer. Chin J Cancer Res 2020;32:115-28.

31. Verma V, Sharma G, Singh A. Immunotherapy in extensive small cell lung cancer. Exp Hematol Oncol 2019;8:5.

32. Ulrich BC, Guibert N. Non-invasive assessment of tumor PD-L1 status with circulating tumor cells. Ann Transl Med 2018;6:S48.

33. Peters S, Creelan B, Hellmann MD, et al. Impact of tumor mutation burden on the efficacy of first-line nivolumab in stage iv or recurrent non-small cell lung cancer: An exploratory analysis of CheckMate 026. Cancer Res 2017;77.

34. Zimmerman S, Das A, Wang S, et al. 2017-2018 Scientific Advances in Thoracic Oncology: Small Cell Lung Cancer. J Thorac Oncol 2019;14:768-83.

35. Boumber Y. Tumor mutational burden (TMB) as a biomarker of response to immunotherapy in small cell lung cancer. J Thorac Dis 2018;10:4689-93.

36. Fenizia F, Pasquale R, Roma C, et al. Measuring tumor mutation burden in non-small cell lung cancer: tissue versus liquid biopsy. Transl Lung Cancer Res 2018;7:668-77.
37. Kim YJ, Keam B, Ock CY, et al. A phase II study of pembrolizumab and paclitaxel in patients with relapsed or refractory small-cell lung cancer. Lung Cancer 2019;136:122-8.

38. Cristescu R, Mogg R, Ayers M, et al. Pan-tumor genomic biomarkers for PD-1 checkpoint blockade-based immunotherapy. Science 2018;362:eaar3593.

39. Ott PA, Bang YJ, Piha-Paul SA, et al. T-Cell-Inflamed Gene-Expression Profile, Programmed Death Ligand 1 Expression, and Tumor Mutational Burden Predict Efficacy in Patients Treated With Pembrolizumab Across 20 Cancers: KEYNOTE-028. J Clin Oncol 2019;37:318-27.

40. Lim JU, Yoon HK. Potential predictive value of change in inflammatory cytokines levels subsequent to initiation of immune checkpoint inhibitor in patients with advanced non-small cell lung cancer. Cytokine 2021;138:155363.

41. Hardy-Werbin M, Rocha P, Arpi O, et al. Serum cytokine levels as predictive biomarkers of benefit from ipilimumab in small cell lung cancer. Oncoimmunology 2019;8:e1593810.

42. Muppa P, Parrilha Terra SBS, Sharma A, et al. Immune Cell Infiltration May Be a Key Determinant of LongTerm Survival in Small Cell Lung Cancer. J Thorac Oncol 2019;14:1286-95.

43. Sonehara K, Tateishi K, Komatsu M, et al. Lung immune prognostic index as a prognostic factor in patients with small cell lung cancer. Thorac Cancer 2020;11:1578-86.

44. Lu Y, Jiang J, Ren C. The clinicopathological and prognostic value of the pretreatment neutrophil-tolymphocyte ratio in small cell lung cancer: A meta-analysis. PLoS One 2020;15:e0230979.

45. Shen XB, Wang Y, Shan BJ, et al. Prognostic Significance of Platelet-To-Lymphocyte Ratio (PLR) And Mean Platelet Volume (MPV) During Etoposide-Based FirstLine Treatment In Small Cell Lung Cancer Patients. Cancer Manag Res 2019;11:8965-75.

46. Wang W, Hodkinson P, McLaren F, et al. Small cell lung cancer tumour cells induce regulatory $\mathrm{T}$ lymphocytes, and patient survival correlates negatively with FOXP3 + cells in tumour infiltrate. Int J Cancer 2012;131:E928-37.

47. Hamilton $G$, Rath B. Immunotherapy for small cell lung cancer: mechanisms of resistance. Expert Opin Biol Ther 2019;19:423-32.

48. Doyle A, Martin WJ, Funa K, et al. Markedly decreased expression of class I histocompatibility antigens, protein, and mRNA in human small-cell lung cancer. J Exp Med 1985;161:1135-51. 
49. Yazawa T, Ito T, Kamma H, et al. Complicated mechanisms of class II transactivator transcription deficiency in small cell lung cancer and neuroblastoma. Am J Pathol 2002;161:291-300.

50. Chen $P$, Zhang L, Zhang W, et al. Galectin-9-based immune risk score model helps to predict relapse in stage I-III small cell lung cancer. J Immunother Cancer 2020;8:e001391.

51. Voong KR, Feliciano J, Becker D, et al. Beyond PD-L1 testing-emerging biomarkers for immunotherapy in nonsmall cell lung cancer. Ann Transl Med 2017;5:376.

52. Hamid O, Schmidt H, Nissan A, et al. A prospective phase II trial exploring the association between tumor microenvironment biomarkers and clinical activity of ipilimumab in advanced melanoma. J Transl Med 2011;9:204.

53. Bridge JA, Lee JC, Daud A, et al. Cytokines, Chemokines, and Other Biomarkers of Response for Checkpoint Inhibitor Therapy in Skin Cancer. Front Med (Lausanne) 2018;5:351.

54. Rizvi NA, Hellmann MD, Snyder A, et al. Cancer immunology. Mutational landscape determines sensitivity to PD-1 blockade in non-small cell lung cancer. Science 2015;348:124-8.

55. Klempner SJ, Fabrizio D, Bane S, et al. Tumor Mutational Burden as a Predictive Biomarker for Response to Immune Checkpoint Inhibitors: A Review of Current Evidence. Oncologist 2020;25:e147-59.

56. Garon EB, Hellmann MD, Rizvi NA, et al. Five-Year Overall Survival for Patients With Advanced NonSmallCell Lung Cancer Treated With Pembrolizumab: Results From the Phase I KEYNOTE-001 Study. J Clin Oncol 2019;37:2518-27.

57. Middleton G, Brock K, Savage J, et al. Pembrolizumab in patients with non-small-cell lung cancer of performance status 2 (PePS2): a single arm, phase 2 trial. Lancet Respir Med 2020;8:895-904.

58. Sabari JK, Leonardi GC, Shu CA, et al. PD-L1 expression, tumor mutational burden, and response to immunotherapy in patients with MET exon 14 altered lung cancers. Ann Oncol 2018;29:2085-91.

59. Donnem T, Hald SM, Paulsen EE, et al. Stromal CD8+ T-cell Density-A Promising Supplement to TNM Staging in Non-Small Cell Lung Cancer. Clin Cancer Res 2015;21:2635-43.

60. Mazzaschi G, Madeddu D, Falco A, et al. Low PD-1 Expression in Cytotoxic CD8(+) Tumor-Infiltrating Lymphocytes Confers an Immune-Privileged Tissue Microenvironment in NSCLC with a Prognostic and Predictive Value. Clin Cancer Res 2018;24:407-19.

61. Ozawa Y, Amano Y, Kanata K, et al. Impact of early inflammatory cytokine elevation after commencement of PD-1 inhibitors to predict efficacy in patients with nonsmall cell lung cancer. Med Oncol 2019;36:33.

62. Hirashima T, Kanai T, Suzuki H, et al. The Levels of Interferon-gamma Release as a Biomarker for Non-smallcell Lung Cancer Patients Receiving Immune Checkpoint Inhibitors. Anticancer Res 2019;39:6231-40.

63. Karachaliou N, Gonzalez-Cao M, Crespo G, et al. Interferon gamma, an important marker of response to immune checkpoint blockade in non-small cell lung cancer and melanoma patients. Ther Adv Med Oncol 2018;10:1758834017749748.

64. Sanmamed MF, Perez-Gracia JL, Schalper KA, et al. Changes in serum interleukin-8 (IL-8) levels reflect and predict response to anti-PD-1 treatment in melanoma and non-small-cell lung cancer patients. Ann Oncol 2017;28:1988-95.

65. Boutsikou E, Domvri K, Hardavella G, et al. Tumour necrosis factor, interferon-gamma and interleukins as predictive markers of antiprogrammed cell-death protein-1 treatment in advanced non-small cell lung cancer: a pragmatic approach in clinical practice. Ther Adv Med Oncol 2018;10:1758835918768238.

66. Costantini A, Julie C, Dumenil C, et al. Predictive role of plasmatic biomarkers in advanced non-small cell lung cancer treated by nivolumab. Oncoimmunology 2018;7:e1452581.
Cite this article as: Lim JU, Kang HS. A narrative review of current and potential prognostic biomarkers for immunotherapy in small-cell lung cancer. Ann Transl Med 2021;9(9):809. doi: 10.21037/atm-21-68 